\title{
Application of Fuzzy-AHP method in the evaluation of logistics capability in e-commerce environment ${ }^{*}$
}

\author{
YANG Lei ${ }^{1,2}$, ZHANG Yi-zhen ${ }^{1}$ \\ (1. Business School, Agricultural University of Hebei, Baoding 071001, China; \\ 2. Economic Trade Department, Hebei College of Finance, Baoding 071051, China)
}

\begin{abstract}
In order to solve the complex problem of evaluation of logistics capability in e-commerce environment, an index system model of logistics capability is established based on the method of Fuzzy-AHP in this paper. The results show that the method of Fuzzy-AHP is a powerful and flexible multi-criteria decision-making tool for dealing with complex problems where both qualitative and quantitative aspects need to be considered, and the human preference model is uncertain and decision-makers are reluctant or unable to assign exact numerical values to the comparison judgments. The evaluation model of logistics capability based on method of Fuzzy-AHP can help to make an effective decision in the evaluation of logistics capability. By this way we can effectively deal with the uncertainty and vagueness in the evaluation process of logistics capability. Logistics companies can diagnose their own weaknesses in the area of logistics and enhance their competitiveness.
\end{abstract}

Key words: Fuzzy-AHP; e-commerce; logistics capability

\section{Introduction}

In today's highly competitive environment, many companies are entering the global arena to gain market share. The cost of logistics and transportation has a large impact on a company's profitability. Global competition in the twenty-first century is forcing companies around the world to reexamine their logistics operations and systems with the objective of reducing costs and improving customer service. E-commerce has brought new opportunities, as well as challenges to logistics management. E-commerce will open an entirely new market for actors in the logistics field. Logistics and distribution systems that function efficiently and effectively in all respects will be crucial for the success of the companies involved. Logistics can be defined as an operational process that includes inputting, storing, transporting and distributing physical goods. E-logistics is an internet-enabled logistics value chain designed to offer competitive logistics services including public warehousing, contract warehousing, transportation management, distribution management, freight consolidation. E-logistics consists of four important components: one-stop value-added services, management of electronic

\footnotetext{
* Acknowledgment: The author would like to thank the anonymous reviewers for their valuable comments and suggestions, which are very helpful in improving the paper. This research was supported by Social Science Foundation of Hebei Province (HB09BYJ050), Major Planning Project of Social Science of Baoding (200901009) and Youth Foundation of Hebei College of Finance (JY200910).

YANG Lei, Ph.D. candidate, Business School, Agricultural University of Hebei, teaching assistant, Economic Trade Department, Hebei College of Finance; research fields: agricultural economy and management, logistics and supply chain management.

ZHANG Yi-zhen, Ph.D., professor, Business School, Agricultural University of Hebei; research fields: agricultural economy and management.
} 
information, a transportation network and automation in warehousing operations. The objectives of e-logistics are reducing operating costs, meeting product delivery deadlines and improving customer services. Nowadays, competitive advantage is often determined by the logistics capability of an organization's supply chain. As a result, it has become an increasingly important management activity. But the evaluation process of the logistics capability is complex. Much of the data are difficult to obtain and ambiguous or vague to interpret. Many companies are using logistics software and the Internet to run their businesses more efficiently and to meet the needs of customers (Aldin \& Stahre, 2003). However, there are also many problems in the modern logistics.

There are many studies performed on logistics capability and Fuzzy-AHP method, such as: Michigan State University Global Logistics Research Team (referred to as MSUGL-RT) (1995) (Weber, et al., 2009) identified 17 types of common logistics capability from 32 possible elements of logistics capabilities through a survey of 111 companies which come from 17 different countries of North America, Europe and the Pacific Basin. Morash, Droge and Vickery (1996) determined eight kinds of logistics capability for the company's strategy: pre-sale customer service, after-sales customer service, delivery speed, delivery reliability, responsiveness of the target market, wide distribution coverage (availability), optional distribution area and low cost distribution. Gimnez and Ventura (2003) pointed out that information and communication technology are very important on the development of supply chain. That means supply chain management (SCM) requires internal and external integration. They analyzed the relationship between internal and external integration processes their effect on firms' performance and their contribution to the achievement of a competitive advantage. Performance improvements are analyzed through costs, stock out and lead time reductions. And, the achievement of a better competitive position is measured by comparing the firm's performance with its competitors' performance. Qureshi, Kumar and Kumar (2009) used the Fuzzy analytic hierarchy process (Fuzzy-AHP) approach to support a generic logistics benchmarking process. Logistics critical success factors from the literature have been identified and prioritized. Using these critical success factors, performance levels of the LOGINET, a 3PL services provider based in western part of India was benchmarked along with four other services providers.

However, these scholar's study are mainly qualitative description, few of empirical research, especially the study combined with e-commerce environment are very little. In short, there is not a scientific and comprehensive assessment system and few scientific and effective methods can be used to evaluate the logistics capability of companies in the e-commerce environment. This implies that manufacturing companies, especially logistics companies, must identify and create effective logistics solutions and establish a scientific evaluation system of logistics capability as soon as possible in order to compete on the marketplace.

This paper proposes an index system model of logistics capability in e-commerce environment and analyzes it by the method of Fuzzy-AHP. It is organized as follows: Section two establishes a logistics capability index system model; Section three evaluates it based on the method of Fuzzy-AHP; Section four comes into the conclusion.

\section{Establishing the logistics capability index system model in e-commerce environment}

Before establishing the logistics capability index system model, we must set up an evaluation index system first. Table 1 illustrates an evaluation index system of logistics capability. 
Application of Fuzzy-AHP method in the evaluation of logistics capability in e-commerce environment

Table 1 Evaluation index system of logistics capability in e-commerce environment

\begin{tabular}{|c|c|c|c|}
\hline & The first level & The second level & The third level \\
\hline \multirow{26}{*}{$\begin{array}{l}\text { Evaluation index system } \\
\text { of logistics capability in } \\
\text { e-commerce environment }\end{array}$} & \multirow{4}{*}{ Finance } & \multirow{2}{*}{ Income } & Interest in net profit margin \\
\hline & & & Sales growth \\
\hline & & Cost & Logistics cost rate \\
\hline & & Investment & ROI \\
\hline & \multirow{5}{*}{ Customer } & \multirow{3}{*}{ End customers } & Customer growth \\
\hline & & & Customer retention \\
\hline & & & Per customer profit contribution \\
\hline & & \multirow{2}{*}{ Suppliers } & Supplier satisfaction \\
\hline & & & Development rate of suppliers \\
\hline & \multirow{12}{*}{ Internal business processes } & \multirow{3}{*}{ Procurement } & Accuracy of the procurement plan \\
\hline & & & Procurement cost control \\
\hline & & & Procurement time control \\
\hline & & \multirow{4}{*}{ Inventory } & Total inventories \\
\hline & & & Order fill rate \\
\hline & & & Inventory turnover \\
\hline & & & Out rate \\
\hline & & \multirow{5}{*}{ Distribution } & $\begin{array}{l}\text { Number of units responsible for customer } \\
\text { distribution centers }\end{array}$ \\
\hline & & & Lift capacity \\
\hline & & & Time arrival rate \\
\hline & & & Delivery time \\
\hline & & & Breakage rate \\
\hline & \multirow{5}{*}{ Learning and growth } & \multirow{3}{*}{ Staff } & The average staff training time \\
\hline & & & Staff satisfaction \\
\hline & & & Staff knowledge level \\
\hline & & Information capacity & IT usage \\
\hline & & Innovation & Innovative transformation of the revenue \\
\hline
\end{tabular}

\section{Evaluation of logistics capability based on Fuzzy-AHP in e-commerce environment}

Analytic hierarchy process (AHP) is a method for ranking decision alternatives and selecting the best one when the decision maker has multiple criteria. This method was first presented by Saaty (1980). With AHP, the decision maker selects the alternative that best meets his or her decision criteria developing a numerical score to rank each decision alternative based on how well each alternative meets them. The AHP is a powerful and flexible multi-criteria decision-making tool for dealing with complex problems where both qualitative and quantitative aspects need to be considered. So, AHP has been widely used as a useful multiple criteria decision making (MCDM) tool or a weight estimation technique in many areas, such as selection, evaluation, planning and development, decision making, forecasting, and so on. In general, evaluation and calculation in AHP can be divided into four stages: scoring the alternatives under each criterion, weighting the criterion, calculating the final score and rank and final decision.

Though the purpose of AHP is to capture the expert's knowledge, the conventional AHP still cannot reflect the human thinking style. In many practical cases, the human preference model is uncertain, and decision-makers might be reluctant or unable to assign exact numerical values to the comparison judgments. Therefore, a method based on Fuzzy-AHP can help to make an effective decision. By this way, we can deal with the uncertainty and 
vagueness in the decision process.

The Fuzzy-AHP technique can be viewed as an advanced analytical method developed from the traditional AHP. Despite the convenience of AHP in handling both quantitative and qualitative criteria of multi-criteria decision making problems based on decision makers' judgments, fuzziness and vagueness existing in many decision-making problems may contribute to the imprecise judgments of decision makers in conventional AHP approaches (Benyoucef \& Canbolat, 2007). In more complex systems, the experiences and judgments of human are represented by linguistic and vague patterns. Therefore, a much better representation of this linguistics can be developed as quantitative data. This type of data set is then refined by the evaluation methods of Fuzzy set theory.

(1) Define decision criteria in the form of a hierarchy of the assessment index of logistics capacity

The hierarchy is structured on different levels: from the top through intermediate levels to the lowest level. We can know from Table 1 that various indicators of the logistics capacity are divided into three levels. The first level is defined as $U=(U 1, U 2, U 3, U 4)$. The next levels are expressed as $U i=(u i 1, u i 2, \ldots, u i j), i=1,2, \ldots, m ; j=$ $1,2, \ldots, n$.

(2) Determine the index weight set

"Expert Choice" as a tool for crisp AHP can determine the contribution of each ratio in creating inconsistency and the ratio with the most contribution. Using AHP, starting from the research objectives, the authors select twenty senior experts which are good at logistics, supply chain management, e-commerce and so on. Give them the forms of consultation, and repeatedly, sought the comments, then construct the evaluation matrixes of logistics capability.

Firstly, weight the criteria, sub-criteria and alternatives as a function of their importance for the corresponding element of the higher level. For this purpose, AHP uses simple pair-wise comparisons to determine weights and ratings, so that the analyst can concentrate on just two factors at one time. Construct each level of the evaluation matrixes of logistics capability. Determine the order and relative weights of each element in each level by constructing the comparative matrixes and the mathematical methods. The expert scoring method is mainly used in this step.

$$
\begin{gathered}
c=\left(c_{i j}\right), \quad i=1,2, \ldots n, \quad j=1,2, \ldots n, c_{i j}=c_{i} / c_{j} \\
\mathrm{C}=\left[\begin{array}{cccc}
\mathrm{c}_{11} & \mathrm{c}_{12} & \ldots & \mathrm{c}_{1 \mathrm{n}} \\
\mathrm{c}_{21} & \mathrm{c}_{22} & \ldots & \mathrm{c}_{2 \mathrm{n}} \\
\ldots & \ldots & \ldots & \ldots \\
\mathrm{c}_{\mathrm{m} 1} & \mathrm{c}_{\mathrm{m} 2} & \ldots & \mathrm{c}_{\mathrm{mn}}
\end{array}\right]
\end{gathered}
$$

In the matrix, $C_{i j}$ means $C_{i}$ relative importance of $C_{j}$. It is described by "1-9 scaling" (Cebeci, 2009) (see Table 2).

Table 2 1-9 Scaling

\begin{tabular}{|c|c|c|c|c|c|}
\hline$C_{i}$ Relative importance of $C_{j}$ & Equal & Fairly strong & Strong & Very strong & Absolute strong \\
\hline$C_{i j}$ & 1 & 3 & 5 & 7 & 9 \\
\hline
\end{tabular}

Secondly, after a judgment matrix has been developed, a priority vector to weight the elements of the matrix is calculated. This is the normalized eigenvector of the matrix. Calculate the matrix structure to derive the greatest characteristic root: 


$$
\lambda_{\max }=\sum_{i=1}^{n} \frac{(B \bar{W})_{i}}{n W_{i}}=\sum_{i=1}^{n} \frac{(B W)_{i}}{n W_{i}}
$$

Then, find the eigenvalues and eigenvectors, and test the consistency. After a good comparison matrix structure, the authors obtained the relative weight matrix factors: $w=\left(w_{1}, w_{2}, w_{3}, w_{4}\right)^{T}$, where, $w_{i}$ is the influence weight of the $i$ kind of factors. Then test the consistency of the matrix. At first, calculate the index of deviating from the consistency, as $C I=\frac{\lambda_{\max }-n}{n-1}$, find out the index of average random consistency $R I$, and then calculate the proportion of consistency $C R=\frac{C I}{R I}$. If $C R<0.1$, it means that the matrix has satisfying consistency (Yang, 2009). Finally, we can gain the weight results by the single order and total order.

(3) Determine the reviews set

The evaluation index system of logistics contains a large number of qualitative indicators that are difficult to quantify. In order to facilitate to evaluate and compare, this article have five (excellent, good, medium, poor, bad) reviews set, namely: $V=(V 1, V 2, V 3, V 4, V 5)=$ (excellent, good, medium, poor, bad). Review set is an aggregate from all kinds of possible evaluation results.

(4) Determine the Fuzzy evaluation matrix

To obtain the evaluation set data by Delphi method, the assessment experts give the Fuzzy choices on various indicators. Statistics the evaluation index system of selection results from the experts, and then calculate in accordance with the established Fuzzy model. After finishing the survey results and statistics, we can get Fuzzy evaluation transformation matrix $R$.

$$
R_{k}=\left(r_{i j}\right) n * 5=\left[\begin{array}{cccc}
r_{11} & r_{12} & \ldots & r_{15} \\
r_{21} & r_{22} & \ldots & r_{25} \\
\ldots & \ldots & \ldots & \ldots \\
r_{n 1} & r_{n 2} & \ldots & r_{n}
\end{array}\right]
$$

where $i=1,2, \ldots, n ; n=1,2,3, \ldots ; k=1,2,3,4 ; j=1,2, \ldots, 5$.

\section{(5) Comprehensive evaluation}

Using Fuzzy evaluation method to obtain the comprehensive Fuzzy evaluation set. For example, to evaluate $U 1, U 2, U 3, U 4$, and then get the comprehensive evaluation results $B 1, B 2, B 3, B 4$. Then using the obtained evaluation results, we can get the results $B$ of the first level $U$, which is the total evaluation result. The comprehensive evaluation results are Fuzzy set of the reviews sets. In practice, we can quantitative the elements of the reviews set in order to reflect all the reviews information, such as $V=$ (excellent, good, medium, poor, bad) $=(10,8,6,4,2)$. Then we will obtain the comprehensive evaluation scores of logistics suppliers, according to its sort of e-commerce capabilities to select the best logistics suppliers.

\section{Conclusion}

Using the comprehensive evaluation method of Fuzzy-AHP, not only all factors are considered in the whole evaluation process, but also all the information at all levels of evaluation are kept. The results are in good to reflect the actual situation of logistics capabilities, and can be easily translated into specific points, to facilitate comparison of high and low levels of logistics. Logistics companies can diagnose their own weaknesses in the area of logistics in order to enhance their competitiveness. 


\section{References:}

Aldin, N. \& Stahre, F.. (2003). Electronic commerce, marketing cannels and logistics platform-A wholesaler perspective. European Journal of Operational Research, 144(1), 270-279.

Benyoucef, M. \& Canbolat, M.. (2007). Fuzzy AHP-Based supplier selection in e-procurement. International Journal of Services and Operations Management, 3(3), 172-192.

Cebeci, U.. (2009). Fuzzy AHP-based decision support system for selecting ERP systems in textile industry by using balanced scorecard. Expert Systems with Applications, 36(7), 8900-8909.

Gimnez, C. \& Ventura, E.. (2003). Supply chain management as a competitive advantage in the Spanish grocery sector. The International Journal of Logistic Management, 14(1), 77-88.

Morash, E, Droge, C. \& Vickery, S.. (1996). Boundary spanning interfaces between logistics, production, marketing and NPD. International Journal of Physical Distribution and Logistics Management, 26(8), 43-62.

Qureshi, M. N, Kumar, P. \& Kumar, D.. (2009). Framework for benchmarking logistics performance using Fuzzy AHP. International Journal of Business Performance and Supply Chain Modelling, 1(5), 82-98.

Saaty, T. L.. (1980). The analytic hierarchy process. New York: McGraw-Hill.

Weber, C. L, Hendrickson, C. T, Matthews, H. S, Nagengast, A, Nealer, R. \& Jaramillo, P.. (2009). Life cycle comparison of traditional retail and e-commerce logistics for electronic products: A case study of buy.com. Sustainable Systems and Technology, 5, 1-6.

Yang, L.. (2009). Empirical analysis on the development of agri-food logistics in Hebei province. Co-operative Economy and Science, 8 , 6-8.

(Edited by Ruby and Chris) 